\title{
Predicting Responses to Pregabalin for Painful Diabetic Peripheral Neuropathy Based on Trajectory- Focused Patient Profiles Derived from the First 4 Weeks of Treatment
}

\author{
Roger A. Edwards - Gianluca Bonfanti - Roberto Grugni • \\ Luigi Manca · Bruce Parsons · Joe Alexander
}

Received: June 11, 2018 / Published online: September 11, 2018

(C) The Author(s) 2018

\section{ABSTRACT}

Introduction: Prediction of final clinical outcomes based on early weeks of treatment can enable more effective patient care for chronic pain. Our goal was to predict, with at least 90\% accuracy, 12- to 13-week outcomes for pregabalin-treated painful diabetic peripheral neuropathy (pDPN) patients based on 4 weeks of pain and pain-related sleep interference data.

Methods: We utilized active treatment data from six placebo-controlled randomized controlled trials $(n=939)$ designed to evaluate

Roger A. Edwards and Gianluca Bonfanti are co-first authors.

Enhanced Digital Features To view enhanced digital features for this article go to https://doi.org/10.6084/ m9.figshare.6994544.

Electronic supplementary material The online version of this article (https://doi.org/10.1007/s12325018-0780-3) contains supplementary material, which is available to authorized users.

R. A. Edwards

Health Services Consulting Corporation, 169

Summer Road, Boxborough, MA 01719, USA

G. Bonfanti · R. Grugni · L. Manca

Fair Dynamics Consulting, srl, Via Carlo Farini 5, 20154 Milan, Italy

B. Parsons · J. Alexander $(\square)$

Pfizer Inc, 235 E 42nd St, New York, NY 10017, USA

e-mail: joe.alexander.jr@pfizer.com efficacy of pregabalin for reducing pain in patients with pDPN. We implemented a threestep, trajectory-focused analytics approach based upon patient responses collected during the first 4 weeks using monotonicity, path length, frequency domain (FD), and k-nearest neighbor $(\mathrm{kNN})$ methods. The first two steps were based on combinations of baseline pain, pain at 4 weeks, weekly monotonicity and path length during the first 4 weeks, and assignment of patients to one of four responder groups (based on presence/absence of $50 \%$ or $30 \%$ reduction from baseline pain at 4 and at $12 / 13$ weeks). The third step included agreement between prediction of logistic regression of daily FD amplitudes and assignment made from kNN analyses.

Results: Step 1 correctly assigned 520/939 patients from the six studies to a responder group using a 3-metric combination approach based on unique assignment to a 50\% responder group. Step 2 (applied to the remaining 419 patients) predicted an additional 121 patients, using a blend of $50 \%$ and $30 \%$ responder thresholds. Step 3 (using a combination of FD and kNN analyses) predicted 204 of the remaining 298 patients using the 50\% responder threshold. Our approach correctly predicted $90.0 \%$ of all patients.

Conclusion: By correctly predicting 12- to 13 -week responder outcomes with $90 \%$ accuracy based on responses from the first month of treatment, we demonstrated the value of 
trajectory measures in predicting pDPN patient response to pregabalin.

Trial Registration: www.clinicaltrials.gov identifiers, NCT00156078/NCT00159679/NCT001 43156/NCT00553475.

Funding: Pfizer.

Plain Language Summary: Plain language summary available for this article.

Keywords: Frequency domain; K-nearest neighbor (kNN); Monotonicity; Painful diabetic peripheral neuropathy (pDPN); Pregabalin; Trajectory prediction

\section{PLAIN LANGUAGE SUMMARY}

To help healthcare providers make better choices on treatments, it is important to understand patient characteristics and how early responses to a drug therapy might predict later responses to that same drug. This study used data from several clinical trials that enrolled patients with pain associated with diabetic peripheral neuropathy treated with pregabalin. We assessed the characteristics of the patients who did or did not show a clinically meaningful response to pregabalin as measured by pain reduction after the first month of therapy and at the end of treatment to identify a "trajectory" of their pain response to better predict their outcomes. We were able to correctly predict the outcomes of these patients at 12 or 13 weeks with $90 \%$ accuracy based on the patients' response in the first 4 weeks.

\section{INTRODUCTION}

Prediction of clinical outcomes based on early weeks of drug therapy can lead to the more effective treatment of patients suffering from chronic pain. These patients pose many challenges for clinicians [1], and the effects of inadequate treatment are felt by patients, clinicians, healthcare delivery systems, and other stakeholders affected by an individual's chronic pain [2-4]. Our prior work with patients with painful diabetic peripheral neuropathy (pDPN) being treated with pregabalin suggested that more information might be discovered from the time course of conditions of care for patients and their treatments $[5,6]$.

While time-series data can be rich sources of information that could be especially useful for predicting patient outcomes, these types of data are often challenging to analyze, especially when various covariates (e.g., sleep interference) also change over time in patients with pDPN [7-9] or other chronic pain conditions [10-12]. Some investigators have used multilevel regression modeling (also known as mixed-effects modeling or hierarchical linear modeling) and multilevel structural equation modeling to evaluate between- and withinsubjects effects of pain, pain-related sleep interference (PRSI) responses, and other variables [10-14]. See Supplemental File 6 for more information on this topic.

Our approach was to seek the simpler solutions first before moving toward building a multilevel model or a growth mixture model [15-25] (as described in Supplemental File 6). Instead, we defined four "responder groups" (i.e., non-latent classes) based on the responder status of patients at specific times (i.e., at week 4 and at the end of study). They are non-latent because they are defined a priori based on known characteristics of patients. The goal of our method was to make predictions about the evolution of pain for each patient through assignment to one of the responder groups. As an alternative to starting with a multilevel model, our approach was to evaluate patterns of the actual values of three linear variables for their ability to predict whether a patient belongs to a particular responder group. In terms of these variables, we had previously explored monotonicity and path length because graphic representation of these metrics had implied differences across responder groups. Based on our earlier work [5], we also started with a stepwise approach that was person-centered (e.g., cluster analysis, latent class analysis, and finite mixture modeling, which aim to classify individuals into distinct groups based on individual response patterns) rather than variable-centered (e.g., regression, factor analysis, and structural equation modeling, which describe relationships among variables) [26]. 
This prior work had affirmed the advantages of stepwise approaches over making predictions all at once.

Our goal in the present study was to correctly predict 12- to 13-week responder outcomes in patients with pDPN treated with pregabalin, with at least $90 \%$ accuracy based on 4 weeks of pain and pain-related sleep interference data.

\section{METHODS}

We utilized data from six randomized placebocontrolled trials (RCTs) $(n=939)$ designed to evaluate the efficacy of pregabalin for reducing pain in patients with pDPN (Pfizer label ref.) [27]. The trials were conducted between November 2000 and March 2009 in Asia, Australia, Canada, Europe, Latin America, the Middle East, South Africa, and the United States. Patients received flexible $(150-600 \mathrm{mg} /$ day) or fixed-dose pregabalin $(75,150,300$, or $600 \mathrm{mg} /$ day) or placebo for 5-13 weeks (Table 1). Since the current modeling was a post hoc pooled analysis evaluating response to treatment, patients were required to have four or more data points where they were confirmed taking pregabalin. Each of the six studies [28-33] shared fundamental inclusion criteria, including the requirement for patients to be aged $\geq 18$ years; have a primary diagnosis of pDPN [type 1 or 2 diabetes mellitus with glycated hemoglobin $(\mathrm{HbA} 1 \mathrm{c}) \leq 11 \%$ and painful, distal, symmetrical, or sensorimotor polyneuropathy for $\geq 6$ months]; have an average pain score over a 7-day baseline period of $\geq 4$ [recorded in patients' daily pain diaries on an 11-point numeric rating scale (NRS), where $0=$ no pain and $10=$ worst possible pain]; and have a score $\geq 40 \mathrm{~mm}$ on the $0-100 \mathrm{~mm}$ visual analog scale of the Short-Form McGill Pain Questionnaire at screening and randomization. Patients with creatinine clearance rates $\leq 60 \mathrm{~mL} / \mathrm{min}$ were excluded, as were patients with any conditions that could jeopardize their health or confound assessment of pain due to pDPN.

All studies were conducted in compliance with the ethics principles originating in or derived from the Declaration of Helsinki, internal review board requirements, or Good
Clinical Practice guidelines, and all participants provided written informed consent before participation.

The primary efficacy outcome in each study was change in pain score (on the 0-10 NRS). Pain responders at the $50 \%$ threshold were defined as those with [pain score at baseline - pain score $(t)] /$ pain score at baseline of $\geq 50 \%$ (where $t=$ time at the end of the study). Secondary efficacy outcomes in each study included change from baseline to end of study in PRSI scores derived from daily sleep diaries in which patients rated how much their pain had interfered with their sleep using a 0-10 NRS (where $0=$ pain does not interfere with sleep and $10=$ pain completely interferes with sleep).

We derived two measures that reflected the trajectories of the NRS data during the first 4 weeks. The first measure, monotonicity, is the extent to which the scores are consistently decreasing or increasing during the first 4 weeks of treatment. A value of -1 or +1 means that each of the four points is lower or higher, respectively, than the previous one. Given that there are four points, there are seven possible values for monotonicity $(-1,-0.667,-0.333$, $0,+0.333,+0.667,+1)$. See Supplementary File S1 for more information about monotonicity.

The second measure, path length, is a measure of the extent to which a trajectory has fluctuations over time. It is the sum of the absolute value of the unit change from week to week. For example, if pain starts at rating of 8 and reduces to 6 after the first week, the value of 2 is used for the week 1 change. This is repeated for weeks 2,3 , and 4 . In theory, the maximum path length could be 40 in the first 4 weeks given integer values from $0(4 \times$ a pain score change of 0$)$ to $40(4 \times$ a pain score change from 0 toward 10 or from 10 toward 0 ). The smaller the path length, the more stable the trajectory (with a minimum value of 0 , which indicates an unchanging trajectory), whereas the larger the path length, the wider the range of values covered by the trajectory. See Supplementary File S2 for more information on path length.

In addition to the primary efficacy outcome of being a $50 \%$ pain responder, we defined another measure to use for prediction based 
Table 1 Summary of patients divided by dose

\begin{tabular}{|c|c|c|c|c|c|c|}
\hline \multirow[t]{2}{*}{ 12-/13-weeks RCTs ${ }^{\mathrm{a}}$} & \multicolumn{6}{|c|}{ Pregabalin dose } \\
\hline & $\begin{array}{l}\text { Flexible } \\
\text { dose }^{b}\end{array}$ & $\begin{array}{l}\text { Flexible adjusted } \\
\text { dose }^{c}\end{array}$ & $150 \mathrm{mg} /$ day & $300 \mathrm{mg} / \mathrm{day}$ & $600 \mathrm{mg} / \mathrm{day}$ & Total \\
\hline$n$ & 83 & 193 & 74 & 297 & 292 & 939 \\
\hline$\%$ of total & 8.8 & 20.6 & 7.9 & 31.6 & 31.1 & 100 \\
\hline Females (\%) & $37(44.6 \%)$ & $116(60.1 \%)$ & $33(44.6 \%)$ & $104(35.0 \%)$ & $116(39.7 \%)$ & $\begin{array}{l}406 \\
(43.2 \%)\end{array}$ \\
\hline Males (\%) & $46(55.4 \%)$ & $77(39.9 \%)$ & $41(55.4 \%)$ & $193(65.0 \%)$ & $176(60.3 \%)$ & $\begin{array}{l}533 \\
\quad(56.8 \%)\end{array}$ \\
\hline Age (years), mean (SD) & $61.3(10.3)$ & $57.0(10.0)$ & $57.7(12.4)$ & $60.4(10.0)$ & $58.6(10.3)$ & $\begin{array}{l}59.0 \\
(10.4)\end{array}$ \\
\hline BMI $\left(\mathrm{kg} / \mathrm{m}^{2}\right)$, mean $(\mathrm{SD})$ & $30.2(4.7)$ & $28.0(5.7)$ & $29.3(4.5)$ & $29.7(7.7)$ & $31.5(7.3)$ & $29.9(6.8)$ \\
\hline Normal weight (\%) & $12(14.5 \%)$ & $65(33.7 \%)$ & $15(20.3 \%)$ & $90(30.3 \%)$ & $44(15.1 \%)$ & $\begin{array}{l}226 \\
(24.1 \%)\end{array}$ \\
\hline Overweight (\%) & $29(34.9 \%)$ & $72(37.3 \%)$ & $29(39.2 \%)$ & $91(30.6 \%)$ & $83(28.4 \%)$ & $\begin{array}{l}304 \\
\quad(32.4 \%)\end{array}$ \\
\hline Obese (\%) & $42(50.6 \%)$ & $56(29.0 \%)$ & $30(40.5 \%)$ & $116(39.1 \%)$ & $165(56.5 \%)$ & $\begin{array}{l}409 \\
\quad(43.6 \%)\end{array}$ \\
\hline $\begin{array}{l}\text { Duration of pDPN (years), } \\
\text { mean }(\mathrm{SD})^{\mathrm{d}}\end{array}$ & $5.1(4.3)^{\mathrm{d}}$ & $2.8(1.4)$ & $4.5(3.9)$ & $4.9(4.0)$ & $4.3(3.3)^{\mathrm{d}}$ & $4.2(3.5)^{d}$ \\
\hline
\end{tabular}

$B M I$ body mass index, $n$ number of patients, $p D P N$ painful diabetic peripheral neuropathy, $R C T$ randomized controlled trial, $S D$ standard deviation

a Study 1008-149 [28] conducted Nov 2000-May 2002 in Australia/Europe/South Africa: DB-RCT with 1 week baseline, 1 week dose escalation, 11 weeks maintenance with placebo, pregabalin 150, pregabalin 300, and pregabalin 600 doses. Study 1008-155 (A0081049) [29] conducted Jul 2001-Dec 2002 in Europe: DB-RCT with 1 week baseline, 1-4 weeks dose escalation, 8-11 weeks maintenance with placebo, pregabalin 150-600 flexible dosing, and pregabalin 600 doses. Study A0081030 [30] conducted Jan 2005-Apr 2006 in Asia/Latin America/Middle East: DB-RCT with 1 week baseline, 6 weeks dose escalation, 6 weeks maintenance, 1 week withdrawal with placebo, pregabalin 150-600 flexible dosing (NCT00156078). Study A0081060 [31] conducted Sep 2004-Oct 2005 in US: DB-RCT with 1 week baseline, 1 week dose escalation, 12 weeks maintenance with placebo, pregabalin 600 dose (NCT00159679). Study A0081071 [33] conducted May 2005-May 2007 in US: DB-RCT with 1-2 weeks baseline, 1 week dose escalation, 12 weeks maintenance, 1 week withdrawal with placebo, pregabalin 300, and pregabalin 600 doses (NCT00143156). Study A0081163 [32] conducted Oct 2007-Mar 2009 in Japan: DB-RCT with 1 week baseline, 1 week dose escalation, 12 weeks maintenance, 1 week withdrawal with placebo, pregabalin 300, and pregabalin 600 doses (NCT00553475)

b Patients with 1-4 weeks escalation phase and 8-11 weeks maintenance (Protocol 1008-155)

c Patients with 6 weeks escalation phase and 6 weeks maintenance (Protocol A0081030)

d 38 patients with missing values for duration of pDPN: 21 of them are flexible dose patients, and 17 are $600 \mathrm{mg} / \mathrm{day}$ dose patients

upon being a $30 \%$ pain responder, which is a threshold that has previously been determined to be clinically meaningful [34]. In summary, we started with the following variables for prediction: Pain $_{0}=$ pain at week 0; Pain $_{4}=$ pain at week 4; $\mathrm{PMono}_{4}=$ monotonicity of weekly pain 
from week 0 to week $4 ; \mathrm{PPL}_{4}=$ path length of weekly pain from week 0 to week $4 ; 50 \mathrm{PRS}_{4}=$ pain responder status at $50 \%$ at week 4 ; and 30 $\mathrm{PRS}_{4}=$ pain responder status at $30 \%$ at week 4 .

We then identified four responder groups reflecting responder status at 4 weeks and responder status at 12-13 weeks. The four responder groups are:

- Patients who were non-responders both at week 4 and at final week;

- Patients who were non-responders at week 4, but then became responders at final week;

- Patients who were responders both at week 4 and at final week; and

- Patients who were responders at week 4 , but then became non-responders at final week.

In order to define the responder groups, we fixed the pain responder thresholds to either 50 or 30\% depending on the different steps of our approach (described below). Thus, in every step of our approach, there were always four responder groups, but each step had its own definition of responder groups based on the pain responder thresholds used. We were then able to use the first 4 weeks of data to predict 12to 13-week responder status without including any additional patient characteristics.

Figure 1 summarizes our three-step approach. In Step 1, we used the actual values of four different variables that, when combined, create a unique pattern (or signature) that can be associated with one of the four responder groups The data elements we used were $\mathrm{PMono}_{4}, \mathrm{PPL}_{4}, \mathrm{Pain}_{4}$, and Pain 0 . We then used unique patterns of three of the four variables (referred to as "3-metric combinations") in order to identify subgroups of patients. Thus, the four possible 3-metric combinations of the data elements were (1) $\mathrm{PMono}_{4}-\mathrm{PPL}_{4}-\mathrm{Pain}_{4},(2)$ $\mathrm{PMono}_{4}-\mathrm{PPL}_{4}-\mathrm{Pain}_{0}$, (3) $\mathrm{PMono}_{4}-\mathrm{Pain}_{4}-\mathrm{Pain}_{0}$, and (4) $\mathrm{PPL}_{4}$-Pain 4 -Pain 0 . We considered all four of these combinations for each patient. In particular, for each patient for whom there was at least one 3-metric combination of the data elements that uniquely fit into only one responder group at week 4 , we made the prediction that the patient would belong to that very responder group at the $50 \%$ responder threshold level at the end of the final week (week 12 or 13, depending on the study). For any patients whom we could not correctly classify in Step 1, we applied Step 2 (subdivided into parts a and b). For Step $2 \mathrm{a}$, we relaxed the responder threshold criterion for prediction in the final week from $50 \%$ to $30 \%$, but maintained the $50 \%$ threshold for week 4 . For Step $2 \mathrm{~b}$, we also relaxed the responder threshold for prediction in the final week to $30 \%$; however, in this case, we additionally relaxed the responder threshold to $30 \%$ for week 4 . Only patients whom we could not correctly predict in Step 2 a moved on to be evaluated in Step $2 b$.

All patients not correctly predicted in the first two steps were then considered in Step 3. In Step 3, we relied on comparing the results of predictions by two different methods. The first method utilized the frequency domain (FD) instead of the typical time domain. While the time domain reflects the changes in the amplitude of a signal over time, the FD shows how many amplitude values of a signal occur within a given time period (i.e., their frequencies). Typically, a transformation is required to make this change, and we applied the widely used Fourier transform. Fourier transformation [35] was applied to the 28 daily pain scores from week 0 to week 4 , and then a logistic regression was performed on the outputs of the Fourier transformation. These outputs included the amplitudes of each of the frequency components (i.e., harmonics) of the signal [36]. With this new way of describing a patient's trajectory, we believed we could identify a patient's responder status at the final week based on the patient's responder status at week 4 . In order to know how to classify the patients, we used the logistic regression of the amplitudes of the patients who had been predicted with 100\% accuracy in Step 1. The hypothesis behind our application of FD analysis is that pain score trajectories that fluctuate with harmonics of similar frequencies may be considered similar. See Supplementary File S3 for more information on the FD analysis.

For the second method, we used a technique called k-nearest neighbors $(\mathrm{kNN})[37,38] . \mathrm{kNN}$ is a classification method that considers each patient as a vector of a certain number of variables, and it associates each patient to other patients "closest" to him or her, where "closest" 


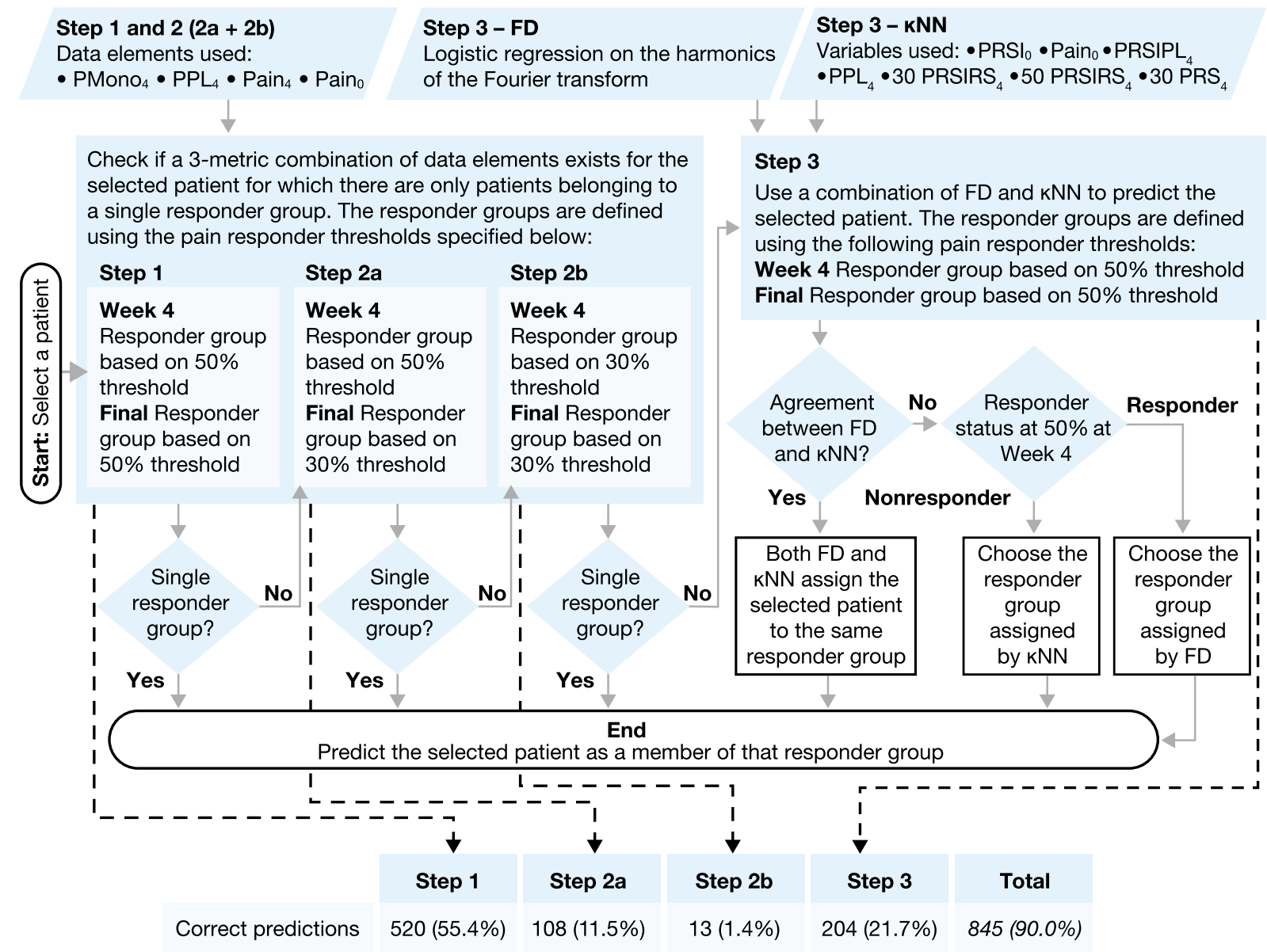

is to be identified with respect to the Euclidean distance (ordinary straight-line distance between two points) computed between the vectors representing the patients. We introduced patient responses for sleep interference from week 0 to week 4 , which conveyed useful information not previously used in our approach thus far in order to better describe patients and, therefore, correctly identify similar patients for prediction. We performed a $\mathrm{kNN}$ analysis (with $k=1$, which means the single closest patient) using the following variables: pain-related sleep interference at week $0\left(\mathrm{PRSI}_{0}\right)$, pain at week $0\left(\right.$ Pain $\left._{0}\right)$, path length of weekly sleep interference from week 0 to week 4 $\left(\mathrm{PRSIPL}_{4}\right), \mathrm{PPL}_{4}$, pain-related sleep interference responder status of $30 \%$ at week 4 (30 PRSIRS $_{4}$ ), pain-related sleep interference responder status of $50 \%$ at week 4 (50 PRSIRS $_{4}$ ), and a pain responder status of $30 \%$ at week $4\left(30 \mathrm{PRS}_{4}\right)$. We added pain-related sleep interference for the $\mathrm{kNN}$ analysis because of the well-documented interactions between pain and PRSI [7-9]. For each patient considered in Step 3, we selected the closest (nearest neighbor) patient based on the $\mathrm{kNN}$ method and assigned the predicted outcome of the patient being considered to be the same as that of the nearest neighbor. We again chose to use the patients correctly predicted in Step 1 as the patients whom we would consider as candidates for the "nearest neighbor". Once we identified the nearest neighbor, we chose this nearest neighbor's outcome to be the predicted outcome for each patient being considered in Step 3. Supporting information on the kNN analysis is available in Supplementary File S4. In essence, by evaluating patients based on a vector of these trajectory-defined pain and PRSI data elements, we generated a patient profile based on trajectory-focused data elements. 
4 Fig. 1 Flow chart of steps for prediction of responder status at 12 or 13 weeks. FD frequency domain, $k N N$ k-nearest neighbor, $\mathrm{Pain}_{0}$ pain at week $0, \mathrm{Pain}_{4}$ pain at week $4, \mathrm{PMono}_{4}$ monotonicity of weekly pain from week 0 to week $4, P P L_{4}$ path length of weekly pain from week 0 to week 4,30 $P_{R S_{4}}$ pain responder status at $30 \%$ at week $4, P R S I_{0}$ pain-related sleep interference at week $0, P R S I P L_{4}$ path length of weekly sleep interference from week 0 to week $4,30 P_{R S I R S_{4}}$ pain-related sleep interference responder status of $30 \%$ at week 4, 50 PRSIRS $_{4}$ pain-related sleep interference responder status of $50 \%$ at week 4 . The specific actions associated with the steps in the flow chart are shown below: (1) Collect two data elements at baseline and weekly until week 4 so that four data points exist for each patient: Pain on 0-10 NRS and pain-related sleep interference on 0-10 NRS. (2) Calculate monotonicity for the first 4 weeks (see Supplemental File 1). (3) Calculate path length for the first 4 weeks (see Supplemental File 2). (4) Generate the following four combinations of three of the data elements generated in the prior three actions: (a) 4-week monotonicity, 4-week path length, pain score at week 4 (PMono $4^{-}$ $\mathrm{PPL}_{4}$-Pain 4 ), (b) 4-week monotonicity, 4-week path length, pain score at baseline (PMono4-PPL $-\mathrm{Pain}_{0}$ ), (c) 4-week monotonicity, pain score at week 4, pain score at baseline (PMono $\left.-\mathrm{Pain}_{4}-\mathrm{Pain}_{0}\right)$, and (d) 4-week path length, pain score at week 4, pain score at baseline ( $\mathrm{PPL}_{4}$ - $\left.\mathrm{Pain}_{4}-\mathrm{Pain}_{0}\right)$. (5) Check four patterns and see if the pattern aligns with those that are uniquely associated with one of the four responder groups (responders at both week 4 and the final week, non-responders at both week 4 and the final week, responders at week 4 but non-responders at the final week, non-responders at week 4 but responders at the final week). (6) If the pattern aligns, predict patient outcome at the final week (Step 1). (7) If the pattern does not align, move to Step 2a and check whether the pattern aligns with those uniquely associated with one of the four responder groups when the 30\% threshold for being a responder in the final week is used. (8) If the pattern aligns with those uniquely associated with one of the four responder groups, then predict the patient's outcome at the final week (Step 2a). (9) If the pattern does not align, move to Step $2 \mathrm{~b}$ and check whether the pattern aligns with those uniquely associated with one of the four responder groups when the $30 \%$ threshold is used for being a responder in the final week and in week 4. (10) If the pattern aligns with those uniquely associated with one of the four responder groups, then predict the patient's outcome at the final week (Step 2b). (11) If the pattern does not align, move to Step 3 and implement the kNN analysis (see Supplemental File 4) by considering the following seven data elements for describing each patient: (a) pain-related sleep interference at baseline, (b) pain score at baseline, (c) 4-week path length of pain-related sleep interference, (d) 4-week path length of pain, (e) pain-related sleep interference responder status at week 4 ( $30 \%$ threshold), (f) pain-related sleep interference responder status at week 4 (50\% threshold), (g) pain responder status at week 4 (30\% threshold). (12) Identify if there are one or more nearest neighbors; if there is only one neighbor with the same vector values, then use it to predict the patient's outcome and if there is more than one neighbor with the same value, the majority is selected for the prediction (see Supplemental File 4 for examples). (13) Before selecting the final choice of outcome for Step 3, also implement the FD analysis. For the FD analysis, use 28 days of daily pain score data and follow the steps outlined in Supplemental File 3. (14) Compare the outcomes predicted by the FD analysis with the outcome predicted by the kNN analysis. If the both the FD and $\mathrm{kNN}$ analyses assign the patient to the same responder group, select that responder group for the outcome. (15) If the responder group assignment differs between the FD analysis and the kNN analysis, use the responder group based on the one assigned by the FD analysis if the patient was a responder at week 4; use the responder group based on the one assigned by the $\mathrm{kNN}$ analysis if the patient was a non-responder at week 4. (16) If daily data are not available, use the kNN analysis alone for Step 3

With respect to the $\mathrm{FD}$ and $\mathrm{kNN}$ analysis methods, we made no a priori assumptions regarding the superiority of one over the other; we considered each independently and looked at agreements and disagreements in their responder predictions for each analysis. Accordingly, we examined two possible cases for each patient: the two methods provided the same prediction (i.e., agreement), or they provided different predictions (i.e., disagreement). In the case of agreement, we decided to trust the prediction for that patient, whereas in the case of disagreement, we evaluated whether in a relative sense one method was superior to the other in identifying patients who were either non-responders at week 4 or responders at week 4. As Step 3 always provided a prediction, there were no patients for whom we did not have a prediction, unlike the previous steps.

We evaluated the overall performance of our approach using an accuracy ratio defined as the proportion of correctly predicted patients, independent of the thresholds used to define the responder groups. 


\section{RESULTS}

Using the various 3-metric combinations approach for unique assignment to a responder group, Step 1 correctly assigned 520 (55.4\%) of the patients from the six studies $(n=939)$ where the pain responder threshold was set to $50 \%$ at week 4 and at the final week. Detailed prediction results by responder group for Step 1 are shown in Supplementary Table S1. Given the 11-point NRS pain scale, there were only seven possible values for Pain $_{0}$, since patients were not enrolled in the studies unless the baseline pain value was at least 4 . There were 11 possible values for Pain $_{4}$, seven possible values for $\mathrm{PMono}_{4}$, and 41 theoretically possible values for $\mathrm{PPL}_{4}$. In reality, no patient had a path length that exceeded 21 . The actual values and patterns of 3-metric combinations of pain data elements are shown in Supplementary File S5.

Step 2 was then applied to the 419 patients remaining after Step 1 (44.6\% of the total of 939 patients). Step 2 added 121 patients (12.9\% of the total of 939 patients) to the number of correctly predicted patients for a total of 641 correctly predicted patients after Steps 1 and 2 (68.3\% of the total of 939 patients). Specifically, Step 2a yielded 108 correct predictions using the 3-metric combination approach with the pain responder threshold set to $50 \%$ at week 4 , with a 30\% threshold for correct prediction at the final week. Step $2 \mathrm{~b}$ yielded 13 correct predictions using the 3-metric combination approach, with the pain responder threshold set to $30 \%$ at week 4 and at the final week. Detailed prediction results by responder group for Step 2 are shown in Supplementary Table S2.

After Steps 1 and 2, there were 298 patients $(31.7 \%)$ remaining who were not uniquely assigned to any responder group. Step 3 correctly predicted 204 patients $(21.7 \%$ of the total of 939 patients) using the $50 \%$ threshold both for responder status at week 4 and at the final week, but did not correctly predict the remaining 94 patients $(10.0 \%$ of the total of 939 patients). There was agreement between the FD analysis and the kNN analysis for most of the predictions in Step 3 (261 out of 298 or $87.6 \%$ ). For the 37 patients where there was disagreement, the best correct prediction was provided by $\mathrm{kNN}$ for the patients who were non-responders at week 4 , while the best correct prediction was provided by FD for the patients who were responders at week 4 . Step 3 results by responder group are shown in Supplementary Table S3. We achieved the best performance (90.0\% correct prediction) when we used kNN for patients who were non-responders at week 4 and FD for patients who were responders at week 4 . However, the differences between FD and $\mathrm{kNN}$ analyses were relatively small for other scenarios. For example, using the same method for both responders and non-responders, the overall percentages of correct predictions were $89.2 \%$ when that method was FD and $89.8 \%$ when the method was kNN. With converse application of the two methods for the handling of disagreements (i.e., FD for patients who were non-responders at week 4 and $\mathrm{kNN}$ for patients who were responders at week 4), the overall correct predictions were $89.0 \%$.

Overall, our approach was able to correctly predict 845 patients $(90.0 \%$ of the total of 939 patients). Most of the 94 incorrectly predicted patients belonged to the responder groups associated with patients who changed their pain responder status between week 4 and the final week: 63 patients were non-responders at week 4 but became responders at the final week, and 20 were responders at week 4 but became non-responders at the final week. Figure 1 illustrates the correct predictions at each step, and the figure legend lists the specific actions associated with each step.

\section{DISCUSSION}

Our finding that we could correctly predict 90\% of the 12- to 13-week responder outcomes in patients by using only 4 weeks of pain and painrelated sleep interference data supports the predictive value of initial responses to pregabalin treatment-especially when the trajectory-related data elements are utilized (e.g., weekly monotonicity and weekly path length for pain scores). By using unique combinations of these data elements, we could achieve a specific signature for over half $(55.4 \%)$ of the 
patients. In essence, these patients resembled each other in terms of subgroups belonging to the same responder group. This "three-dimensional" view of an individual patient's trajectory characteristics provided an appropriate amount of similarity to differentiate groups without requiring more variables that could potentially lead to an excess of small patient groups. No other variables were needed for these patients, which further supports the predictive importance of trajectory information. When we relaxed the responder threshold to 30\% in Steps $2 \mathrm{a}-\mathrm{b}$ (which is still considered a meaningful clinical change), we could correctly predict over two-thirds $(68.3 \%)$ of the pregabalin treatment outcomes in patients with pDPN without adding any other variables. These results also reinforce the importance of a stepwise approach in which simpler techniques are used whenever possible before introducing more complex multilevel modeling techniques.

For the remaining $31.7 \%$ (298) patients whose response we could not predict with the above approaches, we found that FD-based analyses with daily data, specifically amplitudes of harmonic components, contained relevant information that could be utilized effectively; and we used only one basic element of the many FD measures that could have been used. Our results suggest the importance of exploring the use of FD approaches for trajectory analyses, since characterization of these frequency-related features have not been utilized as commonly in non-cardiac medical prediction as they have in other application areas.

The use of kNN machine learning is gaining more widespread use-again supporting the growing perceived value of patient groups derived from how similar they are to one another. Our "nearest neighbors" again reflected trajectory-derived data elements of weekly pain and PRSI rather than other patient variables, further highlighting the predictive gains that become possible when trajectory-focused data elements are considered and analyzed.

By employing the FD analyses separately from the kNN analyses in Step 3, we were able to utilize two different techniques to identify a patient's responder group. The agreement of these two approaches about the responder group provided reinforcing information that could then be utilized for correct prediction of another 181 (19.3\%) patients in Step 3. However, there is further room for improvement with each technique, if correctly predicting $>90 \%$ of the patients is a goal. The finding that neither method was notably more predictive than the other in non-responders versus responders also suggests that both methods demonstrate a persuasive appeal for greater use in prediction. The FD analyses highlight what may be possible with daily pain data and the $\mathrm{kNN}$ analyses underscore what is possible when weekly PRSI measures are added.

The results also motivate us to consider other research and clinical implications. For example, for treatment of pDPN, pregabalin is recommended to be titrated from a lower dose of $150 \mathrm{mg} /$ day (in three divided doses) to $300 \mathrm{mg} /$ day after 1 week [27]. Some physicians may consider a lack of pain response during this titration period to be a reason to discontinue treatment. However, these results provide potentially useful trajectory-based data on patients who were non-responders at week 4 but became responders at end of treatment. Indeed, in a prior study evaluating pooled data for weekly response patterns in pregabalin for neuropathic pain, most of the subjects who became $30 \%$ or $50 \%$ responders at end of treatment achieved their first responder status by weeks 3 or 4 , while a small percentage still showed their first response during weeks 5 and 6 [39]. An improved understanding of these patient profiles could better inform treatment decisions. In addition, since smartphones and wearable mobile technologies provide the potential to easily collect daily data, future studies could examine how daily and weekly pain and PRSI scores recorded during the first month of a pregabalin prescription (e.g., through a smartphone app that could be developed) could be more efficiently utilized as trajectory information to optimize clinical study designs as well as clinical care of patients with pDPN. Clearly, more research is needed; however, the advantages observed in these simple analyses (vs. complex, multilevel, or growth mixture modeling approaches) are evident. Others have noted the usefulness of daily 
diaries [12, 40-43], and the FD analyses also showed the value of daily diary data during the first month of treatment.

Finally, these results reinforce the importance of responses during the first 4 weeks of treatment. Prior work showed that most of the treatment responses occurred during the first 3-4 weeks of treatment [39]. Earlier work has also shown the importance of appropriate dose titration during the first 4 weeks [6].

\section{Limitations}

One limitation is that we only used data from 939 patients from six RCTs designed to study the effects of one medication, pregabalin, in one patient type, patients with pDPN. The robustness of the approach will need further evaluation, since the extent to which the success was affected by these specific trial characteristics is unknown. Also, the circumstances of the individual RCTs might have affected the quality of the data and their utility in the approach. For example, our model included both patients treated with flexible dosing (29.4\% of the total) and those who had fixed dosing (70.6\% of the total), and the impact of these different titration methods on outcomes would need further investigation. However, we consider these variabilities in dosing to be more reflective of real-world practice. Moreover, the pooling of these trials allowed us to create a large dataset for a robust analysis. Next, we further analyzed the 94 patients (10\%) whose responses we could not correctly predict and found that 70 of 94 patients $(75 \%)$ were from fixed-dose arms in the clinical trials. This finding warrants further investigation to understand why trajectory-focused data elements might produce better chances of correct prediction with flexible dosing.

\section{CONCLUSIONS}

By correctly predicting 12- to 13-week responder outcomes with $90 \%$ accuracy based only on data from the first month of treatment, we demonstrated the predictive value of patient profiles enhanced with trajectory-focused data elements in patients with pDPN being treated with pregabalin. These patients could be grouped based on these trajectory-based data elements, and the unique combinations of the scaler measures were highly predictive for twothirds of the patients. For patients whose responses could not be correctly predicted with the scaler measures, analyses based on the FD and $\mathrm{kNN}$ demonstrated the potential value of these approaches to describe such patients. Further research on other pain treatments in pDPN and other chronic pain syndromes can expand upon these findings.

\section{ACKNOWLEDGEMENTS}

Funding. These analyses were funded by Pfizer, along with the open access and articleprocessing charges for this paper. The sponsor also verified the data for accurate reporting of the numbers, but played no role in the final scientific or clinical interpretations of the data.

Medical Writing Assistance. Editorial support in the form of copy editing and formatting was provided by Ray Beck, Jr., PhD, of Engage Scientific Solutions, and was funded by Pfizer.

Authorship. All named authors meet the International Committee of Medical Journal Editors (ICMJE) criteria for authorship for this article, take responsibility for the integrity of the work as a whole, and have given their approval for this version to be published. All authors had full access to all of the data in this study and take complete responsibility for the integrity of the data and accuracy of the data analysis.

Authorship Contributions. Roger A. Edwards and Joe Alexander, Jr. conceived the study. Roger A. Edwards and Gianluca Bonfanti designed the study and the analyses included in the manuscript. Luigi Manca supervised the statistical analyses. Luigi Manca and Roberto Grugni advised on methods and interpretation of the methods. Bruce Parsons and Joe Alexander provided interpretations of the data 
related to clinical relevance and unmet medical needs. All authors participated in the drafting of the paper and final approval of its content.

Disclosures. These analyses and the RCTs were funded by Pfizer. Joe Alexander is an employee of Pfizer. Bruce Parsons is an employee of Pfizer. Roger A. Edwards is an owner of Health Services Consulting Corporation, a paid consultant by Pfizer in connection with this study and development of the manuscript. Gianluca Bonfanti is an employee of Fair Dynamics Consulting. Roberto Grugni is an employee of Fair Dynamics Consulting. Luigi Manca is an employee of Fair Dynamics Consulting. Fair Dynamics Consulting were paid sub-contractors to Health Services Consulting Corporation in conjunction with this study and development of this manuscript.

Compliance with Ethics Guidelines. This article is based on pooled data derived from previously conducted studies, and does not involve any new studies of human or animal subjects performed by any of the authors. For all source studies, participants provided written informed consent, and all related study protocols were approved by the Institutional Review Boards and Ethics Committees of the investigators. Specific details of the ethics and consent to participate may be found in prior publications of the studies [28-33].

Data Availability. The data that support the findings of this study are not publicly available, but can be requested from the authors with Pfizer's permission.

Open Access. This article is distributed under the terms of the Creative Commons Attribution-NonCommercial 4.0 International License (http://creativecommons.org/licenses/ by-nc/4.0/), which permits any noncommercial use, distribution, and reproduction in any medium, provided you give appropriate credit to the original author(s) and the source, provide a link to the Creative Commons license, and indicate if changes were made.

\section{REFERENCES}

1. Finnerup NB, Attal N, Haroutounian S, et al. Pharmacotherapy for neuropathic pain in adults: a systematic review and meta-analysis. Lancet Neurol. 2015;14:162-73.

2. Borsook D, Kalso E. Transforming pain medicine: adapting to science and society. Eur $\mathrm{J}$ Pain. 2013;17:1109-25.

3. Dansie EJ, Turk DC. Assessment of patients with chronic pain. Br J Anaesth. 2013;111:19-25.

4. Stanos S, Brodsky M, Argoff C, et al. Rethinking chronic pain in a primary care setting. Postgrad Med. 2016;128:502-15.

5. Alexander J, Edwards RA, Savoldelli A, et al. Integrating data from randomized controlled trials and observational studies to predict the response to pregabalin in patients with painful diabetic peripheral neuropathy. BMC Med Res Methodol. 2017;17:113.

6. Alexander J Jr, Edwards RA, Manca L, et al. Dose titration of pregabalin in patients with painful diabetic peripheral neuropathy: simulation based on observational study patients enriched with data from randomized studies. Adv Ther. 2018;35:382-94.

7. Parsons B, Emir B, Knapp L. Examining the tme to improvement of sleep interference with pregabalin in patients with painful diabetic peripheral neuropathy and postherpetic neuralgia. Am J Ther. 2015;22:257-68.

8. Vernon M, Brandenburg N, Alvir J, Griesing T, Revicki D. Reliability, validity, and responsiveness of the daily sleep interference scale among diabetic peripheral neuropathy and postherpetic neuralgia patients. J Pain Symptom Manag. 2008;36:54-68.

9. Vinik A, Emir B, Parsons B, Cheung R. Prediction of pregabalin-mediated pain response by severity of sleep disturbance in patients with painful diabetic neuropathy and post-herpetic neuralgia. Pain Med. 2014;15:661-70.

10. Gerhart J, Burns J, Post K, et al. Relationships between sleep quality and pain-related factors for people with chronic low back pain: tests of reciprocal and time of day effects. Ann Behav Med. 2017;51:365-75.

11. Kothari DJ, Davis MC, Yeung EW, Tennen HA. Positive affect and pain: mediators of the withinday relation linking sleep quality to activity interference in fibromyalgia. Pain. 2015;156:540-6. 
12. Moscou-Jackson G, Finan PH, Campbell CM, Smyth JM, Haythornthwaite JA. The effect of sleep continuity on pain in adults with sickle cell disease. J Pain. 2015;16:587-93.

13. Tighe PJ, Le-Wendling LT, Patel A, Zou B, Fillingim RB. Clinically derived early postoperative pain trajectories differ by age, sex, and type of surgery. Pain. 2015;156:609-17.

14. Bromberg M, Connelly M, Anthony KK, Gil KM, Schanberg LE. Prospective mediation models of sleep, pain, and daily function in children with arthritis using ecological momentary assessment. Clin J Pain. 2016;32:471-7.

15. Thomazeau J, Rouquette A, Martinez V, et al. Predictive factors of chronic post-surgical pain at 6 months following knee replacement: influence of postoperative pain trajectory and genetics. Pain Physician. 2016;19:E729-41.

16. Verkleij SP, Hoekstra T, Rozendaal RM, et al. Defining discriminative pain trajectories in hip osteoarthritis over a 2-year time period. Ann Rheum Dis. 2012;71:1517-23.

17. Shiff NJ, Tupper S, Oen K, et al. Trajectories of pain severity in juvenile idiopathic arthritis: results from the Research in Arthritis in Canadian Children Emphasizing Outcomes cohort. Pain. 2018;159(1):57-66. https://doi.org/10.1097/j.pain. 0000000000001064

18. Rzewuska M, Mallen CD, Strauss VY, Belcher J, Peat G. One-year trajectories of depression and anxiety symptoms in older patients presenting in general practice with musculoskeletal pain: a latent class growth analysis. J Psychosom Res. 2015;79:195-201.

19. Page MG, Katz J, Romero Escobar EM, et al. Distinguishing problematic from nonproblematic postsurgical pain: a pain trajectory analysis after total knee arthroplasty. Pain. 2015;156:460-8.

20. Enthoven W, Koes B, Bierma-Zeinstra S, et al. Defining trajectories in older adults with back pain presenting in general practice. Age Ageing. 2016;45:878-83.

21. Dowsey MM, Smith AJ, Choong PFM. Latent class growth analysis predicts long term pain and function trajectories in total knee arthroplasty: a study of 689 patients. Osteoarthr Cartil. 2015;23:2141-9.

22. Althaus A, Arránz Becker O, Neugebauer E. Distinguishing between pain intensity and pain resolution: using acute post-surgical pain trajectories to predict chronic post-surgical pain. Eur J Pain. 2014;18:513-21.
23. Baron E, Bass J, Murray SM, Schneider M, Lund C. A systematic review of growth curve mixture modelling literature investigating trajectories of perinatal depressive symptoms and associated risk factors. J Affect Disord. 2017;223:194-208.

24. Berlin KS, Williams NA, Parra GR. An introduction to latent variable mixture modeling (part 1): overview and cross-sectional latent class and latent profile analyses. J Pediatr Psychol. 2014;39:174-87.

25. Berlin KS, Parra GR, Williams NA. An introduction to latent variable mixture modeling (part 2): longitudinal latent class growth analysis and growth mixture models. J Pediatr Psychol. 2014;39:188-203.

26. Jung T, Wickrama KAS. An introduction to latent class growth analysis and growth mixture modeling. Soc Personal Psychol Compass. 2008;2:302-17.

27. Pfizer Inc. Lyrica (prescribing information). http:// labeling.pfizer.com/ShowLabeling.aspx?id=561. Accessed 31 Jan 2017.

28. Tölle T, Freynhagen R, Versavel M, Trostmann U, Young J Jr. Pregabalin for relief of neuropathic pain associated with diabetic neuropathy: a randomized, double-blind study. Eur J Pain. 2008;12:203-13.

29. Freynhagen R, Strojek K, Griesing T, Whalen E, Balkenohl M. Efficacy of pregabalin in neuropathic pain evaluated in a 12-week, randomised, doubleblind, multicentre, placebo-controlled trial of flexible- and fixed-dose regimens. Pain. 2005;115:254-63.

30. Hoffman D, Sadosky A, Dukes E, Alvir J. How do changes in pain severity levels correspond to changes in health status and function in patients with painful diabetic peripheral neuropathy? Pain Headache. 2010;149:194-201.

31. Arezzo JC, Rosenstock J, Lamoreaux L, Pauer L. Efficacy and safety of pregabalin $600 \mathrm{mg} / \mathrm{d}$ for treating painful diabetic peripheral neuropathy: a double-blind placebo-controlled trial. BMC Neurol. 2008;8:33.

32. Satoh J, Yagihashi S, Baba M, et al. Efficacy and safety of pregabalin for treating neuropathic pain associated with diabetic peripheral neuropathy: a 14 week, randomized, double-blind, placebo-controlled trial. Diabet Med. 2011;28:109-16.

33. ClinicalTrials.gov. Pregabalin vs placebo in treatment of neuropathic pain associated with diabetic peripheral neuropathy. https://clinicaltrials.gov/ ct $2 /$ show/NCT00143156?term=A0081071\&rank=1. Accessed 29 Aug 2018. 
34. Farrar JT, Young JP Jr, LaMoreaux L, Werth JL, Poole RM. Clinical importance of changes in chronic pain intensity measured on an 11-point numerical pain rating scale. Pain. 2001;94:149-58.

35. Elliott DF, Rao KR. Fast transforms: algorithms, analyses, applications. New York: Academic; 1982.

36. Cox DR. The regression analysis of binary sequences. J R Stat Soc. 1958;20:215-20.

37. Alpaydin E. Introduction to machine learning. 2nd ed. Cambridge: MIT Press; 2010.

38. Bezdek JC. Pattern recognition with fuzzy objective function algorithms. Norwell: Kluwer; 1981.

39. Parsons B, Emir B, Clair A. Temporal analysis of pain responders and common adverse events: when do these first appear following treatment with pregabalin. J Pain Res. 2015;8:303-9.
40. Wilt J, Davin S, Scheman J. A multilevel path model analysis of the relations between sleep, pain, and pain catastrophizing in chronic pain rehabilitation patients. Scand J Pain. 2016;10:122-9.

41. Flink I, Linton S, Pain, Sleep and Catastrophizing: The Conceptualization Matters: Comment on Wilt JA, et al. A multilevel path model analysis of the relations between sleep, pain, and pain catastrophizing in chronic pain rehabilitation patients. Scand J Pain. 2016;10:119-21.

42. Finan PH, Hessler EE, Amazeen PG, Butner J, Zautra AJ, Tennen H. Oscillations in daily pain prediction accuracy. Nonlinear Dyn Psychol Life Sci. 2010;14:27-46.

43. Nes AA, Eide H, Kristjansdottir OB, van Dulmen S. Web-based, self-management enhancing interventions with e-diaries and personalized feedback for persons with chronic illness: a tale of three studies. Patient Educ Couns. 2013;93:451-8. 